
$1 / 59285(2)$ PREPARED FOR THE U.S. DEPARTMENT OF ENERGY,

PPPL-2838

UC 420

PPPL-2838

COMPTON HARMONIC RESONANCES, STOCHASTIC INSTABILITIES, QUASILINEAR DIFFUSION, AND COLLISIONLESS DAMPING

WITH ULTRA-HIGH INTENSITY LASER WAVES

BY

J.M. RAX

April, 1992

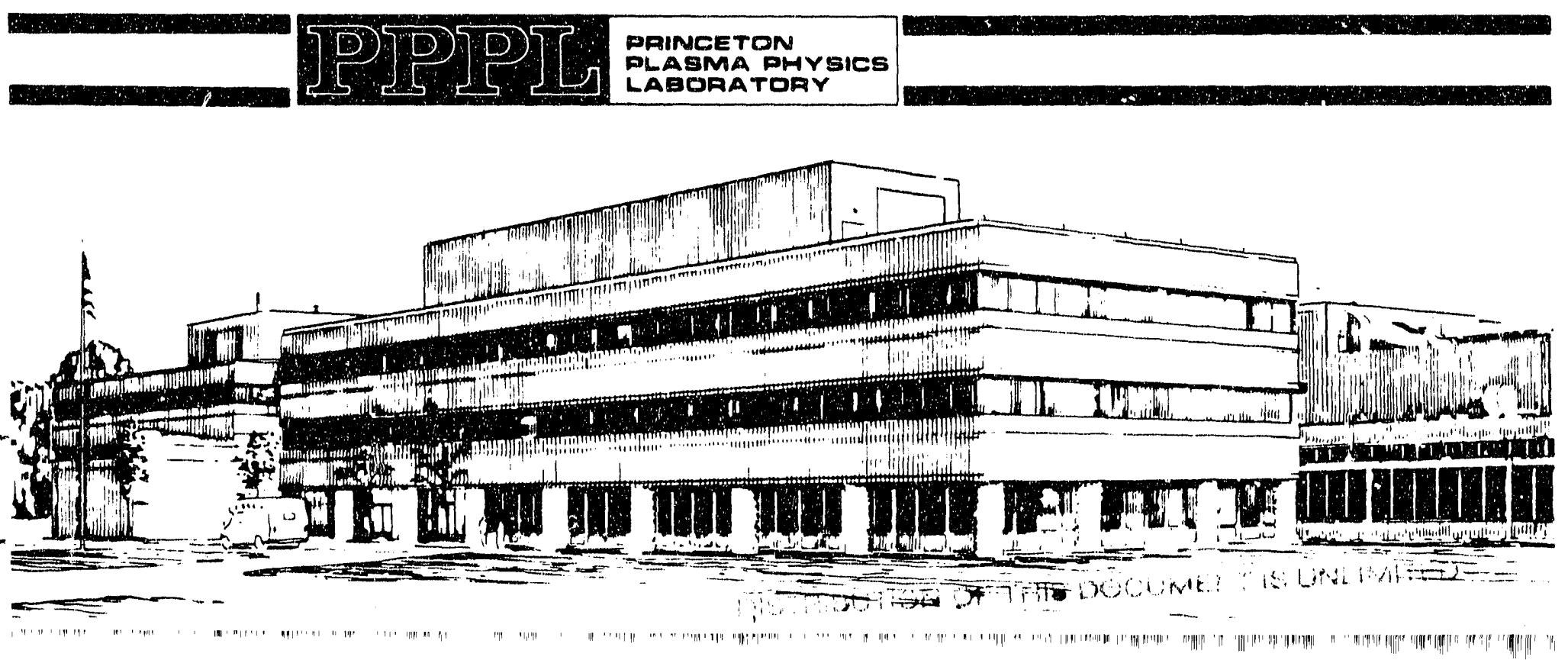




\section{NOTICE}

This report was prepared as an account of work sponsored by an agency of the United States Government. Neither the United States Government nor any agency thereof, nor any of their employees, makes any warranty, express or implied, or assumes any legal liability or responsibility for the accuracy, completeness, or usefulness of any information, apparatus, product, or process disclosed, or represents that its use would not infringe privately owned rights. Reference herein to any specific commercial produce, process, or service by trade name, trademark, manufacturer, or otherwise, does not necessarily constitute or imply its endorsement, recommendation, or favoring by the United States Government or any agency thereof. The views and opinions of authors expressed herein do not necessarily state or reflect those of the United States Government or any agency thereof.

\section{NOTICE}

This report has been reproduced directly from the best available copy.

Available to DOE and DOE contractors from the:

Office of Scientific and Technical Information

P.O. Box 62

Oak Ridge, TN 37831 ;

Prices available from (615) 576-8401.

Available to the public from the:

National Technical Information Service

U.S. Department of Commerce

5285 Port Royal Road

Springfield, Virginia 22161

$703-487-4650$ 


\title{
CONIPTON HARMONIC RESONANCES, STOCHASTIC INSTABILITIES, QUASILINEAR DIFFUSION, AND COLLISIONLESS DAMPING WITH ULTRA- HIGH INTENSITY LASER WAVES
}

J.M. Rax*

Princeton Plasma Physics Laboratory, Princeton University

Princeton N.J. 08540 USA

\begin{abstract}
The dynamics of electrons in two-dimensional, linearly or circularly polarized, ultra-high intensity (above $10^{18} \mathrm{~W} / \mathrm{cm}^{2}$ ) laser waves, is investigated. The Compton harmonic resonances are identified as the source of various stochastic instabilities. Both Arnold diffusion and resonance overlap are considered. The quasilinear kinetic equation, describing the evolution of the electron distribution function, is derived, and the associated collisionless damping coefficient is calculated. The implications of these new processes are considered and discussed.
\end{abstract}




\section{Introduction}

The basic physical processes involved in laser-plasma interaction, up to $10^{17} \mathrm{~W} / \mathrm{cm}^{2}$, are now well understood, on the other hand, a large number of issues remain open in the study of the relativistic interaction regime above $10^{18} \mathrm{~W} / \mathrm{cm}^{2}$.

Most of the theoretical results pertaining to this relativistic regime have been obtained with one dimensional waves, ${ }^{1}$ but little is known about the plasma dynamics in electromagnetic fields which have a two-dimensional structure. Recent advances in pulse compression ${ }^{2}$ now make possible the exploration of laser-plasma interactions, at fluxes above $10^{18} \mathrm{~W} / \mathrm{cm}^{2}$, thus, there is a clear need to identify, and analyze the issues relevant to this ultra-high intensity regime.

This paper addresses this issue of two-dimensional effects and present a general, comprehensible, study of the various stochastic instabilities which come into play when an ultra-high intensity, twodimensional, electromagnetic wave interacts with an electron population.

So far, the only two-dimensional effects which have been predicated are collective processes, such as self focusing. ${ }^{3}$ In self focusing studies, one usualy assumes that the single particle response, to the various waves, is adiabatic, and can be analyzed on the basis of the ponderomotive force arising from the finite transverse size of the light beam. The orbital stochastic instabilities, presented here, are complementary to this adiabatic case, here we identify and study the impact of transverse resonances which break down the adiabaticity.

First, we will identify the Compton harmonic resonances which arise as a result of the nonlinearity of the relativistic orbit. Despite all the studies on spontaneous, and stimulated, Compton scattering, ${ }^{4}$ these resonances are identified here for the first time, because all the previous works were restricted to one dimensional co-propagating waves, or dealed with the the fundamental resonance rather than with the harmonics, or do not consider the regime above $10^{18} \mathrm{~W} / \mathrm{cm}^{2}$. 
Then, we will investigate the stochasticity conditions, derive the quasilinear theory describing the evolution of the electron distribution function, and calculate the associated collisionless damping coefficient. We will carry out this program for linear and circular polarization. Both, the identification of the Compton harmonic resonances (and instabilities), and the derivation of the related kinetic theory, are tne main new results of this paper.

Compton harmonic resonances appears, in the relativistic regime, despite the fact that the waves are travelling at a veiocity far larger than the electron one (they must not be confused with relativisic Landau resonances arising as a result of the beating of several transverse waves), and can be viewed as some kind of cyclotron harmonic resonances between the "figure 8" (in the linear polarization case), or the circular orbit, (in the circular polarization case) nonlinear relativistic orbit, and the laser waves. As in the simple cases of Landau, and cyclotron inieractions, above a given threshold, several of these resonances can overlap, and lead to a stochastic instability. 5

But, for the Compton harmonic case, even in the simple configurations investigated here, the associated dynamical system has $2 \frac{1}{2}$ degrees of freedom, thus the resonant tori are topologically connected in phase space. As a consequence, Arnold diffusion takes place, even for very small perturbations. ${ }^{6}$ Both, Arnold diffusion, and quasilinear diffusion, result in particles stochastic acceleration.

The nonlinearity parameter of an intense electromagnetic wave, with vector potential $\mathrm{A}$, is $\mathrm{eA} / \mathrm{mc}$, $-e$, and $m$ are the electron charge, and mass. In the following we will use $e=m=c=1$.

When $A$ reaches one, $10^{18} \mathrm{~W} / \mathrm{cm}^{2}$ for visible light, the quiver velocity becomes relativistic, nevertheless, the relativistic motion in a one dimensional wave is integrable. ${ }^{7}$ The electron orbit is the combination of an uniform translation with a nonlinear oscillation.

The relativistic effect manifest itself through the anharmonicity of the oscillatory part of the motion, the well known "figure 8" orbit for linear polarization, and the circular orbit for circular 
polarization. In the average rest frame, where the uniform translation cancels, these orbits are depicted on Figs. 1(a), and 2(a), while Figs. 1(b) and 2(b) corresponds to an arbitrary motion.

But, usually, electromagnetic waves are superpositions of several plane waves, with different directions of propagation, thus, the field is intrinsically two-dimensional. Such a generic situation includes the cases of a set of focused beams, a Gaussian beam, or a pump wave with its Raman sidescatters.

In order to understand laser-plasma interaction at intensities above $10^{18} \mathrm{~W} / \mathrm{cm}^{2}$, the very first issue we have to address is the single particle behavior in the fields of these two-dimensional, intense, laser waves.

We will demonstrate that, in two-dimensional, multiple waves, configurations, an irreversible energy transfer, from the waves, to the electrons, can take place as a result of two types of stochastic instabilities: strong, local, instability resulting into quasilinear diffusion, above a given threshold, and weak, widespread, instability, giving rise to Arnold diffusion, regardless to any threshold condition.

This paper is organized as follows. In Secs. 2 and 7 we review the results pertaining to the relativistic orbit in infinite linearly and circularly polarized waves. We cast these results into an Hamiltonian form suitable to a further identification of the Compton resonances. Then, in Sec. 3 (Sec. 8 for circular polarization) we investigate the impact of transverse perturbations propagating at some angle with respect to the one dimensional driving wave considered in Secs. 2 and 7. The Compton harmonic resonances are identified in these sections. In Sec. 4 we describe the various instabilities arising as a result of these resonances. Both Amold diffusion and resonance overlap are considered. The quasilinear kinetic equation describing the evolution of the electron population in the stochastic regime is then derived in Sec. 5 (Sec. 9), and the collisionless damping coefficient due to the transfer of entrgy from the waves to the electrons is calculated in Sec. 6. In Sec. 10 we briefly 
consider the implications of these new instabilities outside the laser-plasma interaction context, i.e., in astrophysics, then we summarize the results and conclude the paper.

\section{Electron orbit in an intense one dimensional linearly polarized wave}

The motion of an electron in a one dimensional, infinite, linearly polarized, wave is integrable. ${ }^{7}$ In this section we will briefly review this important result, and cast it into an Hamiltonian form suitable to a further analysis of the two-dimensional Compton resonances.

Consider a linearly polarized wave, with vector potential $\mathbf{A}$, propagating along the $\mathrm{x}$ axis,

$$
A(\mathbf{r}, t)=A \cos [\Omega(x-t)] \mathbf{e}_{y}
$$

We consider a wave travelling at the velocity of light, in order to isolate pure Compton resonances, not mixed with relativistic Landau processes. In the following calculation we will take $\Omega=1$. The relativistic orbit of an electron interacting with this wave is the well known "figure 8 " motion, drifting in the polarization plane. This orbit is depicted on Fig. 1. As the $z$ degree of freedom will not be excited by the perturbations, the momentum along this $z$ axis is taken to be zero, and will remain unaffected by the resonances and stochastic instabilities investigated in this paper.

To characterize such a driven electron, let us average out the oscillatory part of the motion. The remanning uniform translation is thus described by a parallel momentum, along the wave propagation direction, $x, P_{\|}=\left\langle p_{x}\right\rangle$, a perpendicular momentum, $P_{L}=\left\langle p_{y}\right\rangle$, along the polarization direction, $y$, and a relativistic energy, $E=<\gamma$ ( $\diamond$ stands for the averaging over the oscillations). Note th. th the variables $\mathrm{P}_{\|}, \mathrm{P}_{\perp}, \mathrm{E}$, are not independent and fulfil a dispersion relation, to be given later. 
The interest of these variables, $P_{\|}, P_{\perp}, E$, is twofold, first, they have a clear physical interpretation, second, as we shall see, they provide a simple set of actions to perform the analysis of the instabilities, and to derive the quasilinear kinetic theory.

The motion of an electron in the intense wave Eq. (1) is an Hamiltonian time dependent problem, thus, we have to extend the phase space, in order to get a conservative Hamiltonian system. The time, $t$, will be the additional configuration variable, and minus the relativistic energy, $\gamma$, will be its canonically conjugated momentum. The Hamiltonian is: 8

$$
H_{0}(\mathbf{r}, t, \mathbf{P},-\gamma)=1+\mathbf{p}^{2}-\gamma^{2}=1+[\mathbf{P}+\mathbf{A}(\mathbf{r}, t)]^{2}-\gamma^{2}
$$

$\mathbf{p}$ is the the usual momentum, i.e., the proper time derivative of the position, and $\mathbf{P}$ is the canonical momentum, i.e., $\mathbf{P}=\mathbf{p}-\mathbf{A}$. We can check, with the help of the implicit function theorem, that the orbits on the surface, $\mathrm{H}_{0}=0, \gamma>0$, correspond to the real motion.

$$
\left\{\begin{array}{l}
\frac{\mathrm{d} \mathbf{P}}{\mathrm{dt}}=\frac{\partial \mathrm{H}_{0} / \partial \mathbf{r}}{\partial \mathrm{H}_{\mathrm{o}} / \partial \gamma}=-\frac{\partial \gamma}{\partial \mathbf{r}} \\
\frac{\mathrm{d} \mathbf{r}}{\mathrm{dt}}=-\frac{\partial \mathrm{H}_{\mathrm{o}} / \partial \mathbf{P}}{\partial \mathrm{H}_{\mathrm{o}} / \partial \gamma}=\frac{\partial \gamma}{\partial \mathbf{P}}
\end{array} .\right.
$$

Instead of the configuration, $(\mathbf{r}, t)$, momentum, $(\mathbf{P},-\gamma)$, variables, we will use a set of actions $\left(P_{\perp}\right.$, $\left.\mathrm{P}_{\|}, \mathrm{E}\right)$, angles $(\theta, \varphi, \phi)$. To perform this canonical change of variables we use the generating function $S:^{9}$

$$
S\left(P_{\perp}, P_{\|}, E, x, y, t\right)=P_{\|} x+P_{\perp} y-E t-\frac{P_{\perp} A}{P_{\|}-E} \sin (x-t)-\frac{A^{2}}{8 P_{\|}-8 E} \sin (2 x-2 t)
$$

The momentum, $\mathbf{p}$, can be expressed as, $\mathbf{p}=\mathbf{P}+\mathbf{A}=\partial \mathrm{S} / \partial \mathbf{r}+\mathbf{A}$, so that we recover the usual drifting "figure 8 " solution. 
Note that some textbooks give the nonlinear oscillation solution in the average rest frame, without the complementary drift motion, described by $P_{\|}$and $P_{\perp}$, which is of crucial importance to understand stochastic instabilities, and quasilinear heating, due to Compton harmonic resonances.

With, $p_{x}=\partial S / \partial x=P_{\|}-\left[P_{\perp} A / P_{\|}-E\right] \cos (x-t)-\left[A^{2} / 4 P_{\|}-4 E\right] \cos (2 x-2 t), p_{y}=\partial S / \partial y+A \cos (x-t)$ $=P_{\perp}+A \cos (x-t),-\gamma=\partial S / \partial t=-E+\left[P_{\perp} A / P_{\|}-E\right] \cos (x-t)+\left[A^{2} / 4 P_{\|}-4 E\right] \cos (2 x-2 t)$, it is straightforward to check that, $P_{\|}=\left\langle p_{x}\right\rangle=\left\langle P_{x}\right\rangle, P_{\perp}=\left\langle p_{y}\right\rangle=\left\langle P_{y}\right\rangle, E=\langle\gamma$, as stated previously. Then, after some algebra, the unperturbed Hamiltonian Eq. (2) can be expressed in terms of these actions variables describing the drift of the "figure 8 ".

$$
H_{0}\left(P_{\perp}, P_{\|} E\right)=M^{2}+P_{\|}^{2}+P_{\perp}^{2}-E^{2}
$$

We have introduced the effective mass, $M$, of the electron in the field of the $A$ wave. This effective mass is an important concept of both classical and quantum electrodynamics in strong field. Among other processes, $\mathrm{M}$, is responsible of a nonlinear frequency shift in strong field Compton scattering, and of the enhanced penetration of intense waves in dense plasmas.

$$
M^{2}=1+\frac{A^{2}}{2}
$$

The $P_{\perp}, P_{\|}, E$ variables describe tori in the phase space $(r, t, P,-\gamma)$, or $\left(P_{\perp}, P_{\|}, E, \theta, \varphi, \phi\right)$, and those tori on the upper sheet, $\mathrm{E}>0$, of the hyperboloid, $\mathrm{H}_{\mathrm{o}}=0$, correspond to the real motion. Thus the energy momentum dispersion relation, of an electron in the wave $\mathbf{A}$, writes:

$$
E\left(P_{\|}, P_{\perp}\right)=\sqrt{M^{2}+P_{\|}^{2}+P_{\perp}^{2}} .
$$


Having reviewed this result, pertaining to an ideal infinite one dimensional wave, and cast it into an Hamiltonian problem with the drift variables, we now have to adress the issues of real laser waves which are not one dimensional.

\section{Compton harmonic resonances in an intense linearly polarized wave}

To address these issues, let us consider a generic transverse perturbation, a second wave, $\mathbf{a}$, in the same polarization plane, propagating at some angle, $\alpha$, with respect to $\mathrm{A}$.

$$
a(r, t)=a\left[\cos (\alpha) e_{y^{-}} \sin (\alpha) e_{x}\right] \sin \left[k_{\|} x+k_{\perp} y-\omega t\right]
$$

In the case of intense laser waves packet the frequency of this perturbation will be of the order of the the pump frequency, $\omega \approx \Omega$, and the angle $\alpha$ determined by the propagation direction, $\alpha \approx \operatorname{arctg}\left(k_{\|} / k_{\perp}\right)$. However, in order to make the study quite general, we will not specify the frequency and dispersion properties of this perturbing wave. The Hamiltonian of an electron interacting with both the $\mathbf{A}$ and a waves is:

$$
\mathrm{H}(\mathbf{r}, \mathrm{t}, \mathbf{P},-\gamma)=1+[\mathbf{P}+\mathbf{A}(\mathbf{r}, \mathrm{t})+\mathbf{a}(\mathbf{r}, \mathrm{t})]^{2}-\gamma^{2}=\mathrm{H}_{\mathrm{o}}+2[\mathbf{P}+\mathbf{A}(\mathbf{r}, \mathrm{t})] \cdot \mathbf{a}(\mathbf{r}, \mathrm{t})
$$

As we are performing a perturbative analysis with respect to $\mathbf{a}$, the $\mathbf{a}^{2}$ term is neglected on the right hand side of the second identity. With the help of Eq. (4), we can express this Hamiltonian in terms of the actions $\left(P_{\perp}, P_{\|}, E\right)$, angles $(\theta, \varphi, \phi)$ variables. To carry out this program, we express the old configurations variables as follows: $x=\varphi-\left[P_{\perp} A /\left(P_{\|^{-}} E\right)^{2}\right] \sin (\varphi+\phi)-\left[A^{2} / 8\left(P_{\|}-E\right)^{2}\right] \sin (2 \varphi+2 \phi), y=\theta+$ $\left[A / P_{\|}-E\right] \sin (\varphi+\phi), t=-\phi-\left[P_{\perp} A /\left(P_{\|}-E\right)^{2}\right] \sin (\varphi+\phi)-\left[A^{2} / 8\left(P_{\|}-E\right)^{2}\right] \sin (2 \varphi+2 \phi)$. After some algebra, 
the final result can be expressed as a sum of harmonic interactions weighed by a combination of Bessel functions.

$$
H\left(P_{\perp}, P_{\|}, E, \theta, \varphi, \phi\right)=H_{o}\left(P_{\perp}, P_{\|}, E\right)+a \sum_{N} V_{N}\left(P_{\perp}, P_{\|}, E\right) \cos \left[k_{\|} \varphi+k_{\perp} \theta+\omega \phi+N(\varphi+\phi)\right]
$$

The $\mathrm{N}$ sum is to be taken over all the integer. Note that, for a copropagating wave, $\alpha=\mathrm{k}_{\perp}=0$, and $k_{\|}=\omega$, we recover integrability up to a new canonical transform. The amplitude of the $\mathrm{N}^{\text {th }}$ resonant term is given by:

$$
V_{N}=\sum_{h m n} \delta_{h+m+2 n}^{N} U_{h} J_{m}\left[\frac{A P_{\perp}\left(\omega-k_{\|}\right)}{\left(P_{\|}-E\right)^{2}}+\frac{k_{\perp} A}{P_{\|}-E}\right] J_{n}\left[\frac{A^{2}\left(\omega-k_{\|}\right)}{8\left(P_{\|}-E\right)^{2}}\right]
$$

where the $\mathrm{m}, \mathrm{n}$ sums over the Bessel functions, $\mathrm{J}$, are to be taken over all the integers, and the $\mathrm{h}$ sum is to be estricted to $0, \pm 1, \pm 2 . \delta_{\mathrm{j}}^{\mathrm{i}}$ are the Kroneker symbols. Despite its apparent complexity $\mathrm{V}_{\mathrm{n}}$ can be easily written in terms of the generalized Bessel function, $\mathrm{C}_{\mathrm{N}}$, which has been widely used, and investigated, in the context of strong field quantum electrodynamics. 10

$$
V_{N}=\sum_{|h|=0} U_{h} C_{N-h}\left[\frac{A P_{\perp}\left(\omega-k_{\|}\right)}{\left(P_{\| \mid}-E\right)^{2}}+\frac{k_{\perp} A}{P_{\|}-E},-\frac{A^{2}\left(\omega-k_{\|}\right)}{8\left(P_{\|}-E\right)^{2}}\right]
$$

A review of the basic properties of $C_{N}$, as well as a small argument expansion, relevant to the $A \approx 1$ regime, are given in the appendix. On the other hanc', the $U_{h}$ coupling coefficients involve the wave polarization in a simple way,

$$
U_{h}=2 \delta_{h}^{0}\left[P_{\perp} \cos (\alpha) \cdot P_{\|} \sin (\alpha)\right]+A \delta_{h h \mid}^{1}\left[\cos (\alpha)+\frac{P_{\perp} \sin (\alpha)}{P_{\| \mid}-E}\right]+A^{2} \delta_{|h|}^{2} \frac{\sin (\alpha)}{4\left(P_{\|}-E\right)}
$$


We can try to solve the Hamilton equations associated with Eq. (10) with the standard perturbation technics, i.e., we plug the unperturbed motion in the argument of the perturbating cosines, on the right hand side of Eq. (10).

$$
\left\{\begin{array}{l}
\theta=2 \mathrm{P}_{\perp} \mathrm{s} \\
\varphi=2 \mathrm{P}_{\|} \mathrm{s} \\
\phi=-2 \mathrm{E} s
\end{array}\right.
$$

Where s, the time associated with the extended phase space, is, in fact, two times the proper time of the electron. Such a perturbation scheme fails to converge, because of the occurence of small resonant denominators, when the cosine of the phase remains stationary.

On the basis of Eqs. (10) and (14), this stationary condition gives the Compton harmonic resonances:

$$
k_{\|} P_{\|}+k_{\perp} P_{\perp}-\omega E-N \Omega\left(E-P_{\|}\right)=0
$$

This simple equation is indeed a new result. Before pursuing further the analysis, we must identify which part of the phase space is to be investigated in order to address the issues of high intensity laser plasma interaction in the range $10^{18}-10^{20} \mathrm{~W} / \mathrm{cm}^{2}$. In an experiment, the wave is not infinite, and we are dealing with an initial value problem: a pulse with a front passes an electron at rest, or with a velocity smaller than $c$. This electron enter the wave, and because of the relativistic ponteromotive forces, it picks up an average parallel momentum $P_{\|} \approx O\left[A^{2}\right]$. While in the pulse, the electron behaves as in an infinite wave with an average momentum $P_{\|} \approx O\left[A^{2}\right], P_{\perp} \approx 0$. Thus, we will concentrate mainly on the region $\mathrm{P}_{\|} \approx \mathrm{P}_{\perp} \approx \mathrm{O}[1]$. Note that, if $\omega \neq N \Omega$, no resonances can take place for one dimensional co-propagating waves in vacuum, as the condition $k_{\|} P_{\|}+N \Omega P_{\|}=\omega E$ $+N \Omega E$ can not be fulfilled with the electron dispersion relation Eq. (7), and the wave dispersion relation $k_{\|}=\omega$. On the other hand if $\omega=N \Omega$, this condition is degenerate, as it does not involve the electron action. 


\section{Stochastic instabilities in an intense linearly polarized wave}

In the 6 dimensional phase space $\left(x, y, t, p_{x}, p_{y},-\gamma\right)$, the triplets $\left(P_{\perp}, P_{\|}, E\right)$ label a set of tori. The unperturbed Hamiltonian is non degenerate, thus the Kolmogoroff, Arnold, Moser (KAM) theorem ensures that most of these tori are topologically stable with respect to small perturbations, and are only slightly deformed. ${ }^{5}$ Although a subset, resonant tori defined by Eq. (15), are destroyed by the perturbations. When a $\left(\mathrm{P}_{\|}, \mathrm{P}_{\perp}, \mathrm{E}\right)$ torus fulfil the Eq. (15), the perturbing a wave modify the topology of the trajectories, and induces a trapped domain, between separatrix. The extent of this trapped region is given by the island half-width, in the associated Poincare section.

To calculate the extent of this trapped domain let us consider a resonant torus, $\left(P_{\| c}, P_{\perp c}, E_{c}\right)$, which fulfill the resonance condition Eq. (15). To study the dynamics, in the vicinity of $\left(P_{\| c}, P_{\perp c}\right.$, $E_{c}$ ), we isolate the $\mathrm{N}^{\text {th }}$ resonant interaction, and perform an expansion of the Hamilton equations near $\left(P_{\| c}, P_{\perp c}, E_{c}\right)$. To do so we introduce the reduced action, $J$, and angle, $\psi$.

$$
\left\{\begin{array}{l}
J=\frac{P_{\|}-P_{\| c}}{k_{\|}+N \Omega}=\frac{P_{\perp}-P_{\perp c}}{k_{\perp}}=\frac{E-E_{c}}{\omega+N \Omega} \\
\psi=k_{\|} \varphi+k_{\perp} \theta+\omega \phi+N(\varphi+\phi)
\end{array}\right.
$$

The perturbed action dynamics takes place along the direction of this reduced action. J, and, we end up with a one dimensional nonlinear oscillator.

$$
\left\{\begin{array}{l}
\frac{d J}{d s}=a V_{N}\left(P_{\perp c}, P_{\| c}, E_{c}\right) \sin [\psi] \\
\frac{d \psi}{d s}=\left[2\left(k_{\|}+N \Omega\right)^{2}+2 k_{\perp}^{2}-2(\omega+N \Omega)^{2}\right] J
\end{array}\right.
$$

The trapped domain of this nonlinear oscillator, i.e., the island half-width, $\Delta J$, is given by the square root of the product of the inertia by the nonlinear stiffness. ${ }^{5}$ 


$$
\Delta J=\sqrt{\frac{2 a\left|v_{N}\right|}{\left|k_{\perp}^{2}+\left(k_{\|}-\omega\right)\left(k_{\|}+\omega+2 N\right)\right|}}
$$

From this relation, we immediately obtain the island half-width in action space, as, $\Delta \mathrm{P}_{\|}$ $=\left(k_{\|}+N \Omega\right) \Delta J$, and $\Delta P_{\perp}=k_{\perp} \Delta J$. On the basis of the previous results, if we consider a spectrum of perturbing waves, two kinds of instabilities are to be investigated.

If a $\ll<1$, the Eq. (15) defines a set of 3 dimensional tori, restricted to the 5 dimensional surface of constant energy, Eq. (7), embedded in the 6 dimensional phase space. These resonant tori are associated with separatrix, near which, a thin stochastic layer develops. Even if the width of this stochastic layer is exponentially small, typically $\exp (-\mathrm{B} / \sqrt{\mathrm{aV}})$ with $\mathrm{B}$ of the order of unity, a finite set of waves induces a weak stochastic instability, as a connected web will form on the energy surface, $H_{0}\left(P_{\perp}, P_{\|}, E\right)=0$, depicted on Fig. 3. Arnold diffusion takes place along this web, resulting in the production of a suprathermal population regardless to any threshold condition. 6

If a $\leq 1$, provided the extent of the trapped regions, near these resonant tori, is large enough to allow overlaping, (if the sum of the island half-width becomes of the order of the distance between two nonlinear resonances) a strong, local, stochastic instability appears, resulting in electrons quasilinear diffusion.

The resonance condition, Eq. (15), restricted to the energy surface Eq. (7), can either be considered as a function $P_{\|}\left(\omega, k_{\|}, k_{\perp}, N, P_{\perp}\right)$, or $P_{\perp}\left(\omega, k_{\|}, k_{\perp}, N, P_{\|}\right)$. Two resonances, $(N)$ and $\left(N^{\prime}\right)$, associated with two waves, $\left(\omega, k_{\|}, k_{\perp}\right)$ and $\left(\omega^{\prime}, k_{\|}^{\prime}, k_{\perp}^{\prime}\right)$, overlap if the Chirikov threshold criterion: $\Delta \mathrm{P}_{\perp}+\Delta \mathrm{P}_{\perp}^{\prime}>\mathrm{P}_{\perp}\left(\omega, \mathrm{k}_{\|}, \mathrm{k}_{\perp}, \mathrm{N}, \mathrm{P}_{\|}\right)-\mathrm{P}_{\perp}\left(\omega^{\prime}, \mathrm{k}_{\|}^{\prime}, \mathrm{k}_{\perp}^{\prime}, \mathrm{N}, \mathrm{P}_{\|}\right)$, or $\Delta \mathrm{P}_{\|}+\Delta \mathrm{P}_{\|}^{\prime}>\mathrm{P}_{\|}\left(\omega, \mathrm{k}_{\|}, \mathrm{k}_{\perp}, \mathrm{N}, \mathrm{P}_{\perp}\right)$. $P_{\|}\left(\omega^{\prime}, k_{\|}^{\prime}, k_{\perp}^{\prime}, N, P_{\perp}\right)$, is fulfilled.

A two-dimensional wave packet, propagating along $x$, and diffracting along $y$, can be decomposed as the sum of waves propagating in the $(x, y)$ plane. One can consider the main component of the spectra as a driving wave $\mathrm{A}$, and the other components, propagating at some angle, as a set of perturbing waves a. For example, as a model configuration, we take a driving 
wave, $\mathbf{A}$, and two symmetric a waves, at the same frequency, propagating at some angle $\alpha$, such that $k_{\|}=\Omega \cos (\alpha), k_{\perp}= \pm \Omega \sin (\alpha)$, and $\omega=\Omega$.

Figure 4 displays the Compton resonances in the case, $\alpha=\pi / 4, A=1$. Note that, if $a<<1$, we can identify a resonance with the stochastic layer around the separatrix, and Fig. 4 is, in fact, the Amold stochastic web. The resonances pattern changes dramatically if we take the case $\alpha=3 \pi / 4$, depicted on Fig. 5 , for example as a result of stimulated, backward, Raman sidescattering. As opposed to the previous situation, an accumulation of higher order resonances appears. Thus an electron starting from $\mathrm{P}_{\|} \approx 1 \approx 1, \mathrm{P}_{\perp} \approx 0$ can easily reach the high energy part of the phase space.

On Fig. 4, considering resonance overlap, the most dangerous resonances is clearly $N=-1$. Taking the small argument limit of the generalized Bessel function, 10 to the lowest order in $\mathrm{A}$ and $\alpha$, we obtain the following rough estimate of the Chirikov criterion: $|a A \alpha|>1$. This condition is to be complemented by the one which validates the perturbative calculation $\mathrm{A}>\mathrm{a}$. Thus the final condition writes $A>a>1 / A$, and we conclude that, if $A<1$, it can not be fulfilled, i.e., a twodimensional wave packet, can not induce resonance overlap, below $10^{18} \mathrm{~W} / \mathrm{cm}^{2}$, but, above, if the angular divergence is large enough, the $\mathrm{N}=-1$ resonances of the various components can induce stochastic acceleration.

For the case of Fig. 5, the high energy resonance overlap, seems to be more easy to achieved, but, in the $A \approx 1$ regime, it is to be noted that it is limited by the fact that the distance between the $N$ and $N+1$ resonances, for large $N$, decrease like $N^{-1 / 2}$, although the $V_{N}$ coupling scale as $A^{N} / N$ !. The single particle orbit stability of various two-dimensional wave configurations can be assessed on the basis of Eqs. (15) and (18), but, rather than carrying out such a program, let us address the important issue of the electron population dynamics in the stochastic regime. 


\section{Quasilinear theory in an intense linearly polarized wave}

A kinetic theory describing fast electrons production in the Arnold diffusion case is difficult to set up. On the other hand, in the resonance overlap regime, the quasilinear theory provides a description of the evolution of the electrons distribution function $F\left(P_{\|}, P_{\perp}, t\right)$.

Iir order to derive this Fokker-Planck quasilinear equation describing the dynamics of the electron population, $F\left(P_{\|}, P_{\perp}, t\right)$, interacting with a wave spectra, $a\left(k_{\|}, k_{\perp}\right)$, we start from the Hamilton equations.

$$
\frac{\mathrm{dP}_{\|}}{\mathrm{dt}}=\left.\frac{\partial \mathrm{H} / \partial \varphi}{\partial \mathrm{H}_{0} / \partial \gamma}\right|_{\mathrm{H}_{0}=0}, \quad \frac{\mathrm{dP}_{\perp}}{\mathrm{dt}}=\left.\frac{\partial \mathrm{H} / \partial \theta}{\partial \mathrm{H}_{0} / \partial \gamma}\right|_{\mathrm{H}_{0}=0}
$$

Then, for one wave, and one resonance, we express the increments $\delta P_{\|}$, and $\delta P_{\perp}$, laking place during a small time, $\delta \mathrm{t}$, as a function of the initial phase of the perturbation $\psi_{0}$. Within the framework of the quasilinear approximation, these increments are associated with the slow resonant evolution of $F$, so that we can take $\gamma=E$ and $t=2 E s$, and disregard fast, non resonant, oscillations.

$$
\delta I=\frac{\delta P_{\|}}{k_{\|}+N \Omega}=\frac{\delta P_{\perp}}{k_{\perp}}=\frac{a V_{N}\left(P_{\perp} P_{\|}\right)}{2 E} \frac{\cos \left[\psi_{0}\right]-\cos \left[\psi_{0}+\left(k_{\|} P_{\|}+k_{\perp} P_{\perp}-\omega E-N \Omega\left(E-P_{\|}\right)\right) \delta t / E\right]}{k_{\|} P_{\|}+k_{\perp} P_{\perp}-\omega E-N \Omega\left(E-P_{\|}\right) / E}
$$

In the stochastic regime this phase, $\psi_{0}$, is to be treated as a random variable as a result of the existence of a positive Liapounov exponent. Thus we can annly the "random phase approximation" (RPA) to evaluate the diffusion coefficients on the basis uf Eq. (21).

After some algebra, and the use of the identity $2 \sin ^{2}(u / 2 \varepsilon) / u^{2}=\pi \delta(u) / \varepsilon$, if $\varepsilon=0^{+}$, (which is equivalent to the Landau causal prescription around the Compton pole, if we use the Laplace transform of the Vlasov equation rather than the Hamilton equations) we obtain:

$$
D_{I I}=\frac{\langle\delta I \delta I\rangle}{\delta t}=\pi \frac{a^{2} V_{N}^{2}\left(P_{\perp} P_{\|}\right)}{4 E} \delta\left[k_{\| P_{\|}}+k_{\perp} P_{\perp} \cdot \omega E-N \Omega\left(E-P_{\|}\right)\right]
$$


The bracket $\Leftrightarrow$ stands for the phase average and $\delta\left[k_{\|} P_{\|}+k_{\perp} P_{\perp}-\left(\omega E-N \Omega\left(E-P_{\|}\right)\right]\right.$is the experted Dirac function of the Compton resonance. Then, it is straightforward to write down the Fokker-Planck equation associated with this resonant diffusion process: $\partial F / \partial t=1 / 2[\partial / \partial \mathbf{P}] \cdot \mathbf{D}_{\mathbf{P P}} \cdot[\partial / \partial \mathbf{P}] \cdot F$. Where $D_{P_{\|} P_{\|}}=\left(k_{\| \mid}+N \Omega\right)^{2} D_{I I}, D_{P_{\|} P_{\perp}}=\left(k_{\|}+N \Omega\right) k_{\perp} D_{I I}$, and $D_{P_{\perp} P_{\perp}}=k_{\perp} k_{\perp} D_{I I}$. Finally, we end up with the following quasilinear equation

$$
\frac{\partial F\left(P_{\|}, P_{\perp}, t\right)}{\partial t}=\sum_{N}\left[\left(k_{\|}+N \Omega\right) \frac{\partial}{\partial P_{\|}}+k_{\perp} \frac{\partial}{\partial P_{\perp}}\right] D_{N}\left(P_{\|}, P_{\perp}\right)\left[\left(k_{\|}+N \Omega\right) \frac{\partial}{\partial P_{\|}}+k_{\perp} \frac{\partial}{\partial r_{\perp}}\right] F\left(P_{\|}, P_{\perp}, t\right)
$$

Note that the very same equation can be obtained from a Vlasov representation of the dynamics of $F\left(P_{\|}, P_{\perp}, \theta, \varphi, t\right)$ averaged over $(\theta, \varphi)$. As in the Landau and cyclotron cases, the singular character of the diffusion tensor, i.e., the fact that it is a sum of Dirac function of the Compton resonances , is removed through an integration of $D_{N}$ over the $a\left(k_{\|}, k_{\perp}\right)$ spectra.

$$
D_{N}\left(P_{\|}, P_{\perp}\right)=\frac{\pi}{8} a^{2}\left(k_{\|}, k_{\perp}\right) \frac{V_{N}^{2}\left(P_{\|}, P_{\perp}\right)}{E} \delta\left[k_{\|} P_{\|}+k_{\perp} P_{\perp}-E \omega-N \Omega\left(E-P_{\|}\right)\right]
$$

Thus, in the stochastic regime, the pertubing waves induce a quasilinear stochastic heating of the electron population, $F\left(P_{\|}, P_{\perp}\right)$, and, on $E q .(23)$, we see that this heating takes place along the diffusion paths:

$$
\frac{\mathrm{dP}_{\perp}}{\mathrm{dP}_{\|}}=\frac{\mathrm{k}_{\perp}}{\mathrm{k}_{\|}+\mathrm{N} \Omega}
$$

Equations. (23), (24), and (25) are one of the main results of this paper, they allow to describe the electrons dynamics in the stochastic regirse. In fact, in a collisional plasma, a decorrelation mechanism is provided by the collisions, so that the RPA, which validates this kinetic description, is applicable even below the stochasticity threshold. 
The kinetic evolution of $\mathrm{F}$ can be summarized as follows, as a result of the interaction of the nonlinear resonances, near these resonances, Eq. (15), the distribution function is flattened along the diffusion paths, Eq. (25). This quasilinear flattening of a momentum gradient, of magnitude $P$, takes place on a time scale $D_{N} / P^{2}$, so that, in the regime $A \approx 1, a \leq 1,|N| \approx 1, \omega \approx \Omega$, the relaxation of a momentum gradient of the order of mc occurs on a very short time scale of the order of few $\Omega^{-1}$.

As power is exchanged between the waves and the electrons, we have to address the issue of the wave damping resulting from this irreversible quasilinear transfer of energy.

\section{Collisionless damping in an intense linearly polarized wave}

The collisionless damping process that results from the quasilinear stochastic heating of the electrons, is, in some sense, similar to collisionless cyclotron damping, as the wave phase velocity is always larger than the electron velocity. However, here, we are dealing with a drifting "figure 8" orbit, rather than with a Larmor circular one. Note that the driving wave, $\mathbf{A}$, is not affected, but electrons heating takes place at the expense of the perturbing lateral waves, a. To study this process, let us introduce the density of energy of these waves:

$$
U\left(k_{\|}, k_{\perp}, t\right)=\varepsilon_{o} \omega^{2} a^{2}\left(k_{\|}, k_{\perp}, t\right)
$$

$\varepsilon_{0}$ is the vacuum permitivity, and, for the sake of simplicity, we have considered the vacuum dispersion relation. The energy consevation theorem writes:

$$
\frac{\partial \mathrm{U}\left(\mathrm{k}_{\|}, \mathrm{k}_{\perp}, \mathrm{t}\right)}{\partial \mathrm{t}}+\int \mathrm{d} \mathrm{P}_{\|} \mathrm{dP} \mathrm{P}_{\perp} \mathrm{E}\left(\mathrm{P}_{\|}, \mathrm{P}_{\perp}\right) \frac{\partial \mathrm{F}\left(\mathrm{P}_{\|}, \mathrm{P}_{\perp}, \mathrm{t}\right)}{\partial t}=0
$$

All the factor in the second term of Eq. (27) have been calculated previously. Thus, we use Eq. (23), and, after integrating by part the momentum sum, Eq. (27) becomes: 


$$
\frac{\partial U\left(k_{\|}, k_{\perp}, \mathfrak{t}\right)}{\partial t}=\sum_{N} \alpha_{N}\left(k_{\|}, k_{\perp}\right) U\left(k_{\|}, k_{\perp}, \mathfrak{t}\right)
$$

The sign of $\alpha$ is determined by the derivative of $F$ along the diffusion paths Eq. (25). Needless to say, the sign of this damping coefficient can be either positive or negative, depending on the free energy content of F. For a thermal distribution, we always get damping, but some inverted distribution give rise to kinetic instabilities and wave growth. Such kinetic instabilities are, in fact, nothing but, relativistic, harmonic, stimulated, Compton scattering.

$$
\begin{aligned}
\alpha_{N}\left(k_{\|}, k_{\perp}\right)=\frac{\pi}{4} \frac{\omega_{p}^{2}}{\omega^{2}} \int d P_{\|} d P_{\perp} \frac{\omega+N \Omega}{E} V_{N}^{2}\left(P_{\|}, P_{\perp}\right) \delta\left[k_{\|} P_{\|}+k_{\perp} P_{\perp}-E \omega-N \Omega\left(E-P_{\|}\right)\right] & \\
& {\left[\left(k_{\|}+N \Omega\right) \frac{\partial}{\partial P_{\|}}+k_{\perp} \frac{\partial}{\partial P_{\perp}}\right] \cdot F\left(P_{\|}, P_{\perp}\right) }
\end{aligned}
$$

$\omega_{p}$ is the plasma frequency, and we have used the normalization $\int \mathrm{dP}_{\|} d \mathrm{dP}_{\perp} F=1$. This last equation is ar important result, and provides the optical depth due to the Compton harmonic resonances in ultrahigh intensity laser-plasma interaction problems.

\section{Electron orbit in an intense one dimensional circularly polarized wave}

So far, we have restricted our studies to linear polarization, in fact, all the previous results, on stochastic instabilities and kinetic theory, can be derived for an arbitrary polarization. However, the resulting formulae do not bring out any new physics and look rather cumbersome.

From the experimental point of view, as the previous optical depth is rather small, the observation of the effectiveness of Compton harmonic stochastic heating in the ultra-high intensity regime rely on the observation of the production of fast electrons. Thus we have to answer the following question: 
what is the most efficient wave configuration to observe the Compton harmonic stochastic instabilities and the electron quasilinear stochastic heating with ultra-high intensity laser waves? This question can be answered on the basis of a general study, but, it turns out that the result can be easily found with simple symmetry arguments.

The very reason why a irremovable nonlinear resonance appears in a perturbed integrable system, is a conflict of symmetry between the perturbing Hamiltonian and the unperturbed one. Nœther's theorem ensures integrability as a result of a high degree of symmetry, so that, if a perturbation is invariant under a subgroup of the unperturbed symmetry group, integrability still holds. Broken symmetry results in the appearance of destroyed tori.

If we consider two waves, with two very different polarizations, and directions of propagation, we can expect that a large number of tori will be destroyed. One of the simplest ways to achieve such a configuration, is to consider a circularly polarized driving wave, and a linearly polarized perturbing wave, propagating at right angles. We will prove that this configuration is a very efficient heating scheme, and that the stochasticity threshold, up to $\mathrm{N}=-10$, can easily be reached in the regime $A \approx 1$. One can understand this as follows: in the plane of polarization, the pump wave drives a circular orbit, although in the very same plane the perturbation want to drive a "figure 8". Obviously, the folding and welding of an "O" into an " 8 " imply a change of topology which cannot be achieved perturbatively.

The study of a circularly polarized pump is also very interesting because an intense circularly polarized wave appears in the radiation zone of a pulsar, as a result of the rotation of the dipolar magnetic field of the neutron star. Values in the range $A \approx 10^{11}$ for electrons, and $A \approx 10^{8}$ for protons, have been predicted. We will briefly point out the relevance of the present stochastic acceleration mechanism to pulsar physics, in the last section of this paper. 
The motion of an electron in the field of an infinite one dimensional circularly polarized electromagnetic wave is integrable. Consider such a wave with vector potential:

$$
A(r, t)=A \cos [z-t] e_{x}+A \sin [z-t] e_{y} .
$$

The orbit of an electron is the combination of a uniform translation and a nonlinear circular oscillation, and is depicted on Fig. 2. The relativistic effect manifest itself through the anharmonicity or the circular part of the motion. In the average rest frame, where the uniform translation cancels, the trajectory is a circle in the polarization plane depicted on Fig. 2(a), while Fig. 2(b) corresponds to an arbitrary orbit.

As in the previous case, to find a convenient set of variables, we average out the circular part of the motion. The remaining uniform translation is characterized by a parallel momentum, along the wave propagation direction, $\mathrm{P}_{\|}=\left\langle\mathrm{p}_{z}\right\rangle$, two perpendicular average momentum, $\left\langle\mathrm{p}_{x}\right\rangle$ and $\left\langle\mathrm{p}_{y}\right\rangle$, in the polarization plane, and an average relativistic energy $E=<>$. In fact the momentum along the y axis can be taken equal to zero and remains unaffected by the resonances and stochastic instabilities investigated in the following sections, thus, we define $P_{\perp}=\left\langle p_{x}>\right.$.

In order to use a set of actions, $\left(P_{\perp}, P_{\|}, E\right)$, angles, $(\theta, \varphi, \phi)$, variables, we have to perform a canonical transformation. This is achieved with the help of the generating function $\mathrm{S}:{ }^{9}$

$$
S\left(P_{\perp}, P_{\|}, E, x, z, t\right)=P_{\perp} x+P_{\|} z-E t-\frac{P_{\perp} A}{P_{\|}-E} \sin (z-t) .
$$

To check that this generating function corresponds to the physical definition of the average momentum, we simply use the identities: $p_{x}=\partial S / \partial x+A \cos (z-t)=P_{\perp}+A \cos (z-t), p_{z}=\partial S / \partial z=P_{\|}$ $\left[P_{\perp} A / P_{\|^{-}} E\right] \cos (z-t),-\gamma=\partial S / \partial t=-E+\left[P_{\perp} A / P_{\| \mid}-E\right] \cos (z-t)$. After some algebra, the unperturbed 
Hamiltonian can be expressed in terms of the action variables describing the $x, z$ drift of the circular orbit.

$$
H_{0}\left(P_{\perp}, P_{\|}, E\right)=1+[P+A(r, t)]^{2}-\gamma^{2}=M^{2}+P_{\|}^{2}+P_{\perp}^{2}-E^{2}
$$

This time, the effective mass, $\mathrm{M}$, of an electron in a circularly polarized wave is given by:

$$
M^{2}=1+A^{2}
$$

The $P_{\perp}, P_{\|}$, E variables describe tori in phase space, and those on the upper sheet, $E>0$, of the hyperboloid, $\mathrm{H}_{\mathrm{o}}=0$, correspond to the real motion. The energy momentum dispersion relation is: $E^{2}=M^{2}+P_{\|}^{2}+F_{\perp}^{2}$

\section{Compton resonances and stochastic instabilities in an intense circularly polarized wave}

The analysis of the stability of this drifting circular motion is similar to the linear polarization case. We consider a second wave, a, propagating along the $\mathrm{x}$ axis with a linear polarization.

$$
\mathbf{a}(\mathbf{r}, \mathrm{t})=\mathbf{a} \cos [k x-\omega t] \mathbf{e}_{y}
$$

The interaction Hamiltonian of an electron, in the field of both waves, is: $2[\mathbf{P}+\mathbf{A}(\mathbf{r}, t)] \cdot \mathbf{a}(\mathbf{r}, t)$. With the help of Eq. (31), we can express this Hamiltonian in term of the actions, $\left(P_{\perp}, P_{\|}, E\right)$, angles, $(\theta, \varphi, \phi)$, variables of the unperturbed motion: $x=\theta+\left[A / P_{\|}-E\right] \sin (\varphi+\phi), z=\varphi-\left[A P_{\perp} /\left(P_{\|^{-}}\right.\right.$ $\left.E)^{2}\right] \sin (\varphi+\phi), t=-\phi-\left[A P_{\perp} /\left(P_{\|}-E\right)^{2}\right] \sin (\varphi+\phi)$. The final result writes:

$$
H\left(P_{\perp}, P_{\|}, E, \theta, \varphi, \phi\right)=H_{o}\left(P_{\perp}, P_{\|}, E\right)+a \sum_{N} W_{N}\left(P_{\perp}, P_{\|}, E\right) \sin [k \theta+(1) \phi+N(\varphi+\phi)]
$$


The $\mathrm{N}$ sum is to be taken over all the integer, and the amplitude of the $\mathrm{N}^{\text {th }}$ resonant perturbation can be expressed in terms of the derivative of the Bessel functions $\mathrm{J}_{\mathrm{N}}^{\prime}$.

$$
W_{N}\left(P_{\perp} P_{\|}, E\right)=2 A J^{\prime}\left[\frac{k A}{P_{\|}-E}+\frac{\omega A P_{\perp}}{\left(P_{\|}-E\right)^{2}}\right]
$$

Then, in order to find the re onances, we take the unperturbed phase motion, $\theta=2 \mathrm{P}_{\perp} \mathrm{s}, \varphi=2 \mathrm{P}_{\|} \mathrm{s}$, and $\phi=-2 E s$, and plug it into the arguments of the perturbing sines in Eq. (35). As expected, such a perturbation scheme fails to converge because of the occurrence of small resonant denominators when the sine phase remain stationary.

$$
k P_{\perp}-\omega E-N \Omega\left(E-P_{\|}\right)=0 \text {. }
$$

These are the Compton resonances in a circularly polarized wave. To investigate the small denominator problem, in the vicinity of a resonant point $\left(P_{\| c}, P_{\perp c}, E_{c}\right)$, which fulfill Eq. (37), we introduce the reduced action, J, and angle, $\psi$.

$$
\left\{\begin{array}{l}
J=\frac{P_{\|}-P_{\| c}}{N \Omega}=\frac{P_{\perp}-P_{\perp c}}{k}=\frac{E-E_{c}}{\omega+N \Omega} \\
\psi=k \theta+\omega \phi+N(\varphi+\phi)
\end{array}\right.
$$

The unperturbed Hamiltonian Eq. (32) is non degenerate, thus KAM theorem ensures that most of the $\left(\mathrm{P}_{\perp}, \mathrm{P}_{\|}, \mathrm{E}\right)$ tori are stab!e with respect to small perturbation. Although resonant tori, Eq. (37), are destroyed even by small perturbations. The reduced variables, J, $\psi$, describe the the occurrence of a trapped domain on these resonant tori.

$$
\left\{\begin{array}{l}
\frac{d J}{d s}=a W_{N}\left(P_{\perp c}, P_{\| c}, E_{c}\right) \sin [\psi] \\
\frac{d \psi}{d s}=\left[2(N \Omega)^{2}+2 k^{2}-2(\omega+N \Omega)^{2}\right] J
\end{array}\right.
$$


To assess the potentiality of a strong stochastic instability we calculate the island half-width.

$$
\Delta J=\sqrt{\frac{2 a\left|w_{N}\right|}{\left|k^{2}-\omega^{2}-2 N \omega\right|}}
$$

The interaction between two nonlinear resonances leads to a stochastic instability, provided they overlap, i.e., the sum of the previous half-width is larger than the distance between these two resonances. Let us consider $A=2, a=0.6, k=\omega=\Omega$, and calculate the resonance location, $P_{\| N}$, and island half-width, $\Delta_{\| N}$, along the parallel momentum axis $P_{\perp}=0$, for $-12<N<-2$. The Chirikov stochasticity threshold, is fulfilled when $\Delta_{\| \mathbb{N}}+\Delta_{\| N+1}$ becomes of the order of $P_{\| N+1}-P_{\| N}$. For the first resonances, the following table clearly shows that the stochastic regime is reached, although at higher energy, despite the accumulation of large $\mathrm{N}$ resonances, stocilasticity desapears as a result of the smallness of the island size.

\begin{tabular}{cccccccccccc}
\hline $\mathrm{N}$ & -1 & -2 & -3 & -4 & -5 & -6 & -7 & .8 & .9 & -10 & -11 \\
\hline $\mathrm{P}_{\Perp \mathrm{N}}$ & 0 & 1.29 & 2 & 2.53 & 2.98 & 3.37 & 3.72 & 4.04 & 4.34 & 4.67 & 4.88 \\
\hline$\Delta_{\Perp N}$ & 0.93 & 1.09 & 1.07 & 0.92 & 0.72 & 0.52 & 0.36 & 0.24 & 0.15 & 0.09 & 0.05 \\
\hline
\end{tabular}

Thus, Compton harmonic stochastic heating with two vacuum waves, in the intensity range, $10^{18}-10^{19} \mathrm{~W} / \mathrm{cm}^{2}$, can be achieved with this configuration.

\section{Quasilinear theory and collisionless damping in an intense circularly polarized wave}

Before concluding, for the sake of completeness, we will briefly derive the kinetic theory relevant to this circular polarization case. The action increments, $\delta \mathrm{P}_{\|}$, and $\delta \mathrm{P}_{\perp}$, taking place during a small time, $\delta t$, are obtained from the Harnilton equations associated with Eq. (35). 


$$
\delta \mathrm{I}=\frac{\delta \mathrm{P}_{\|}}{\mathrm{N} \Omega}=\frac{\delta \mathrm{P}_{\perp}}{\mathrm{k}}=\frac{\mathrm{aW} \mathrm{W}_{\mathrm{N}}\left(\mathrm{P}_{\perp} \mathrm{P}_{\|}\right)}{2 \mathrm{E}} \frac{\sin \left[\psi_{0}\right]-\sin \left[\psi_{0}+k \mathrm{P}_{\perp}-\omega \mathrm{E} \cdot \mathrm{N} \Omega\left(\mathrm{E} \cdot \mathrm{P}_{\|}\right) \delta \mathrm{t} / \mathrm{E}\right]}{\mathrm{kP} \mathrm{P}_{\perp}-\omega \mathrm{E} \cdot \mathrm{N} \Omega\left(\mathrm{E} \cdot \mathrm{P}_{\|}\right) / \mathrm{E}}
$$

If the stochasticity criterion is fultilled, the phase $\psi_{0}$ is a random vartable and the quasilinear diffusion coefficient can be calculated with the RPA.

$$
\frac{\langle\delta I \delta I>}{\delta t}=\pi \frac{a^{2} W_{N}^{2}\left(P_{\perp} P_{\|}\right)}{4 E} \delta\left[k P_{\perp-\omega E-N \Omega\left(E-P_{\|}\right)}\right]
$$

Then, on the basis of this diffusion coefficient, we write the Fokker Planck equation, $\partial F / \partial t=$ $1 / 2[\partial / \partial P] . Q_{P P} .[\partial / \partial P] . F\left(Q_{P_{\|} P_{\|}}=N \Omega^{2}<\delta 1 \delta 1>/ \delta t, Q_{P_{\|} P_{\perp}}=N \Omega k<\delta I \delta I>/ \delta t, Q_{P_{\perp} P_{\perp}}=k k<\delta I \delta i>/ \delta t\right)$.

$$
\begin{array}{r}
\frac{\partial F\left(P_{\|}, P_{\perp}, t\right)}{\partial t}=\frac{\pi}{8} a^{2} \sum_{N}\left[N \Omega \frac{\partial}{\partial P_{\|}}+k \frac{\partial}{\partial P_{\perp}}\right] \frac{W_{N}^{2}\left(P_{\|}, P_{\perp}\right)}{E} \delta\left[k P_{\perp}-E \omega-N \Omega\left(E-P_{\|}\right)\right] \\
{\left[N \Omega \frac{\partial}{\partial P_{\|}}+k \frac{\partial}{\partial P_{\perp}}\right] \cdot F\left(P_{\|}, P_{\perp}, t\right) .}
\end{array}
$$

The singular behavior of $Q$ is removed through an integration over the wave spectrum. Quasilinear heating of the population $F$, takes place along diffusion paths whose equation is:

$$
\frac{\mathrm{dP}_{\perp}}{\mathrm{dP}_{\|}}=\frac{\mathrm{k}}{\mathrm{N} \Omega}
$$

Finally, the damping coefficient of the perturbing waves, which is also the growth rate for an inverted population, is obtained on the basis of energy conservation.

$$
\alpha_{N}\left(k_{\|}, k_{\perp}\right)=\frac{\pi}{4} \frac{\omega_{p}^{2}}{\omega^{2}} \int d P_{\|} d P_{\perp} \frac{\omega+N \Omega}{E} W_{N}^{2}\left(P_{\|}, P_{\perp}\right) \delta\left[k P_{\perp}-E \omega-N \Omega\left(E-P_{\|}\right)\right]\left[(N \Omega) \frac{\partial}{\partial P_{\|}}+k \frac{\partial}{\partial P_{\perp}}\right] . F\left(P_{\|}, P_{\perp}\right)
$$

$\omega_{p}$ is the plasma frequency, and we have used the normalization $\int \mathrm{d} \mathbf{P F}=1$. Needless to say, the results of Eqs. (43), and (45) can be obtained from the relativistic Vlasov equation in an intense circularly polarized wave. Clearly, under typical experimental condition, the optical depth associated 
with this collisionless damping is very small, this is mainly due to the fact that ultra high intensity laser waves ( $a \leq 1$ ) are required, thus, even if a cold electron population is significantly heated up, the relative energy loss of the wave remains very small. On the other hand, the production of fast electrons, in the $y$ direction of the proposed configuration, is a clear signature of the new resonant diffusion process described here.

\section{Discussion and conclusion}

The previous circular polar $z$ ation configuration provides one of the simplest and most efficient ways to demonstrate the effectiveness of Compton harmonic stochastic heating with the forthcoming ultra-high intensity laser pulses. Ultra-high intensity waves with circular polarization are also involved in the physics of pulsar environment, so let us briefly discuss stochastic acceleration in this astrophysical situation.

The high energy environment of pulsars as long been recognized as one of the major candidates to to explain the ultra-high energy tail of the cosmic radiation spectrum. The theories of charged particles acceleration can be roughly classified between, non stochastic mecnanisms (where the particle acquires energy at the expense of a coherent electromagnetic structure through a regular interaction), and stochastic acceleration (which are diffusive in nature and result from the sum of a set of incoherent exchanges of energy between the particle and the field).

As far as astrophysical acceleration mechanisms, with pulsar fields, are concerned, only coherent mechanisms have been considered, ${ }^{11}$ surprisingly, the most simple mechanism, a charge interacting with intense electromagnetic waves in vacuum, above the stochasticity threshold, has

never been put forward. For typical pulsation, $10^{-2} \mathrm{~s}$, and magnetic field, $10^{8} \mathrm{~T}$, values in excess of $A \approx 10^{8}$ for a protons are predicted in the wave region of the spinning magnetized neutron star. 
Clearly the stochastic acceleration mechanism presented in this paper is relevant to the physics in the radiation zone. But, with such a high field, radiation reaction is to be considered, thus a slowing down term is to be added to the quasilinear Fokker Planck equation Eq. (43).

To summarize, we have identified the Compton harmonic resonances associated with the electron orbit in a two-dimensional laser waves at intensities above $10^{18} \mathrm{~W} / \mathrm{cm}^{2}$. Then we have demonstrated and discussed the fact that they are the source of two kinds of stochastic instabilities. A weak, widespread, instability, resulting from Arnold diffusion along the stochastic web, and a strong, local, instability, as a result of resonance overlap. In this later situation, quasilinear diffusion along the diffusion paths of Eqs. (25), and (44), takes place according to the kinetic equations (23), and (43). As a consequence of this stochastic heating, the perturbing electromagnetic waves are damped. These processes have been analyzed for various wave polarizations and configurations; they are inoperative below $10^{18} \mathrm{~W} / \mathrm{cm}^{2}$, but will become dominant, in laser-plasina interaction, in the intensity range $10^{18}-10^{20} \mathrm{~W} / \mathrm{cm}^{2}$.

\section{Acknowledgments}

I wish to acknowledge useful discussions with N. Fisch and C. Karney. This work was supported by the U.S Department of Energy under contract number DE-AC02-76-CHO3073. 


\section{Appendix}

\section{Generalized Bessel function}

The generalized Bessel function is a special function which occurs in the study of quantum processes in intense electromagnetic waves. For this reason it has been widely investigated. In this appendix we will review its definition, and its Taylor expansion.

The Function $\mathrm{C}_{\mathrm{N}}$ is usually defined as:

$$
C_{N}(\alpha, \beta)=\sum_{j=-\infty}^{j=+\infty} J_{N+2 j}(\alpha) J_{j}(\beta)=\frac{1}{2 \pi} \int_{-\pi}^{\pi} e^{i \alpha \sin \varphi-i \beta \sin 2 \varphi-i N \varphi} d \varphi
$$

$\mathrm{J}$ are the ordinary bessel function and the denomination of $\mathrm{C}$ come from the obvious identity:

$$
\mathrm{J}_{\mathrm{N}}(\mathrm{z})=\mathrm{C}_{\mathrm{N}}(\mathrm{z}, 0) \text {. }
$$

Two kinds of limits are relevant to the previous studies on stochastic instabilities in an intense wave.

(i) The Taylor expansion, ${ }^{10}$ for small $\alpha$ and $\beta$, is relevant to the situation $A \approx 1$. In order to evaluate Eq. (13) in this regime, for the resonance $N=-1$, we can use:

$$
\left\{\begin{array}{l}
C_{0}(\alpha, \beta)=1-\frac{\alpha^{2}}{4}+\frac{\alpha^{4}}{64}-\frac{\beta^{2}}{4} \\
C_{1}(\alpha, \beta)=\frac{\alpha}{2}+\frac{\alpha \beta}{4}-\frac{\alpha^{3}}{16} \\
C_{2}(\alpha, \beta)=\frac{\alpha^{2}}{8}+\frac{\alpha^{2} \beta}{8}-\frac{\alpha^{4}}{96}-\frac{\beta}{2} .
\end{array}\right.
$$

- (ii) On the other hand, the asymptotic expansion for $\alpha \approx \beta \approx N \approx \infty$, is to be used if one is interested in a quantitative analysis of orbital stability and stochastic acceleration near a pulsar, $\mathrm{A} \geq 10^{8}$. Both uniform and nonuniform asymptotic expansions can be found in Refs. [10]. 


\section{References}

1 P. Sprangle, E. Esarey, and A. Ting, Phys. Rev. Lett. 64, 2011 (1990). P. Sprangle, E. Esarey, and A. Ting, Phys. Rev A 4, 4464 (1990). J.M. Rax, and N.J. Fisch, Phys. Fluids B 4 (to appear may 1992).

${ }^{2}$ M. Pessot, J. Squier, G. Mourou and D.J. Harter, Opt. Lett. 14, 797 (1987). M. Pessot, J. Squier, P. Bado, G. Mourou and D.J. Harter, IEEE J. Quantum Electron. QE-25, 61 (1989).

${ }^{3}$ G.Z. Sun, E. Ott, Y.C. Lee, and P. Guzdar, Phys. Fluids 30, 526 (1987). P. Sprangle, C.M. Tang, and E. Esarey, IEEE Trans. Plasma Sc. 15, 145 (1987). W.B. Mori, C. Joshi, J.M. Dawson, D.W. Forslund, and J.M. Kindel, Phys. Rev. Lett. 60, 1298 (1988). A. Ting, E. Esarey, and P. Sprangle, Phys. Fluids B 2, 1390 (1990).

${ }^{4}$ E.S. Sarachik and G.T. Schappert, Phys. Rev. D 10, 2738, (1970). R.E. Waltz, and O.P. Manley Phys. Fluids 21, 808, (1978). R.H. Pantell, G. Soncini and H.E. Puthoff, IEEE J. Quantum Electron. QE6, 905 (1968). A.T. Lin and J.M. Dawson, Phys. Fluids 18, 201 (1975) A. Hasegawa, K. Mima, P. Sprangle, H.H. Szu, and V.L. Granatstein, App. Phys. Letters 29, 542 (1976). R.C. Davidson, Phys. Fluids 29, 267 (1986).

${ }^{5}$ G. M. Zaslavski and B.V. Chirikov, Sov. Phys. Usp. 14, 549, (1972). B.V. Chirikov, Phys. Rep. 52, 263, (1979). Lichtenberg and Liebermann Regular and Stochastic Motions. (Springer Verlag, New-York, 1983). 
6V.I. Amold, Dokl. Akad. Nauk. SSSR 156, 9 (1964), G.M. Zaslavskii et al Sov. Phys. Usp. 31, 887 (1988). G.M. Zaslavskii et al, W'eak chaos and quasi-regular patterns, (Cambridge University Press, Cambridge, 1991).

7 T.W. Kibble, Phys. Rev. 138, 740 (1965). T.W. Kibble, Phys. Rev. 150, 1060 (1966). J.H. Eberly, Progress in Optics 7, E. Wolf ed., 361 (North Holland, Amsterdam, 1969).

${ }^{8}$ J.D. Jackson, Classical Electrodynamics, $2^{\text {nd }} \mathrm{ed}, 577$ (Wiley, New York, 1975).

${ }^{9}$ L. Landau and E. Lifshitz The Classical Theory of Fields, $4^{\text {nd }}$ ed., 112 (Pergamon Press, Oxford, 1975).

${ }^{10}$ A.I. Nikishov and V.I. Ritus, J.E.T.P 19, 529 (1964). C. Leubner, Phys. Rev. A 23, 2877 (1981). V.I. Ritus, J. Sov. Laser Research 6, 610 (1985).

11 J.E. Gunn, and J.P. Ostriker, Astro. J., 157, 1395 (1969). J.E. Gunn, and J.P. Ostriker, Phys. Rev. Lett., 22, 728 (1969). R. M Kulsrud, J.E. Gunn, and J.P. Ostriker, Phys. Rev. Lett., 28, 636 (1972). 


\section{Figures Caption}

Figure 1: Orbit of an electron in a one dimensional linearly polarized wave.

Figure 2: Orbit of an electron in a one dimensional circularly polarized wave.

Figure 3: Dispersion surface, Compton resonances, and Arnold web, in momentum space.

Figure 4: Compton resonances in the $\left(P_{\|}, P_{\perp}\right)$ plane, $A=1, k_{\mid}=\Omega \sqrt{2} / 2, k_{\perp}= \pm \Omega \sqrt{2} / 2, \omega=\Omega$.

Figure 5: Compton resonances in the $\left(P_{\|}, P_{\perp}\right)$ plane, $A=1, k_{\|}=-\Omega \sqrt{2} / 2, k_{\perp}= \pm \Omega \sqrt{2} / 2, \omega=\Omega$. 


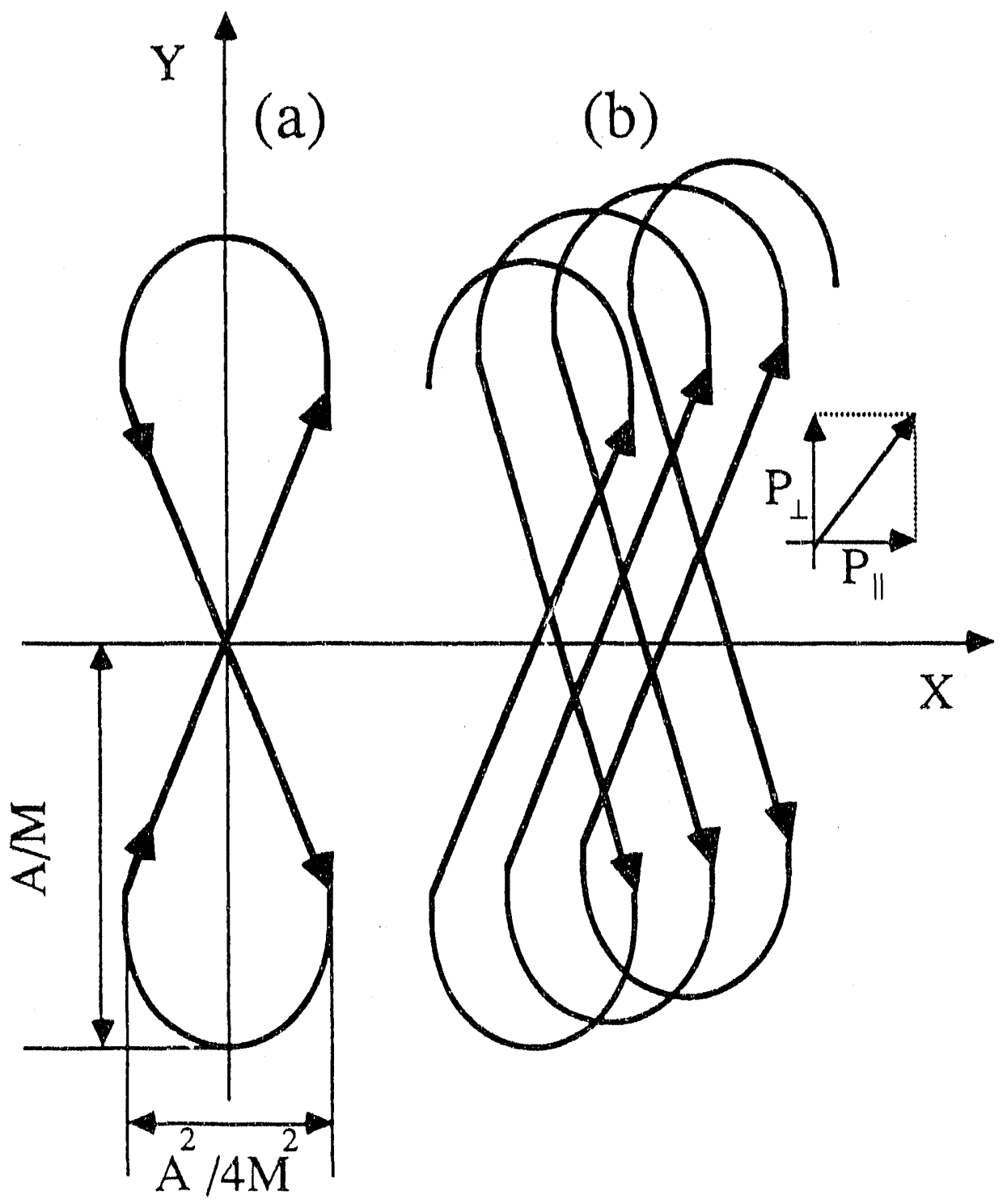

Fig. 1 


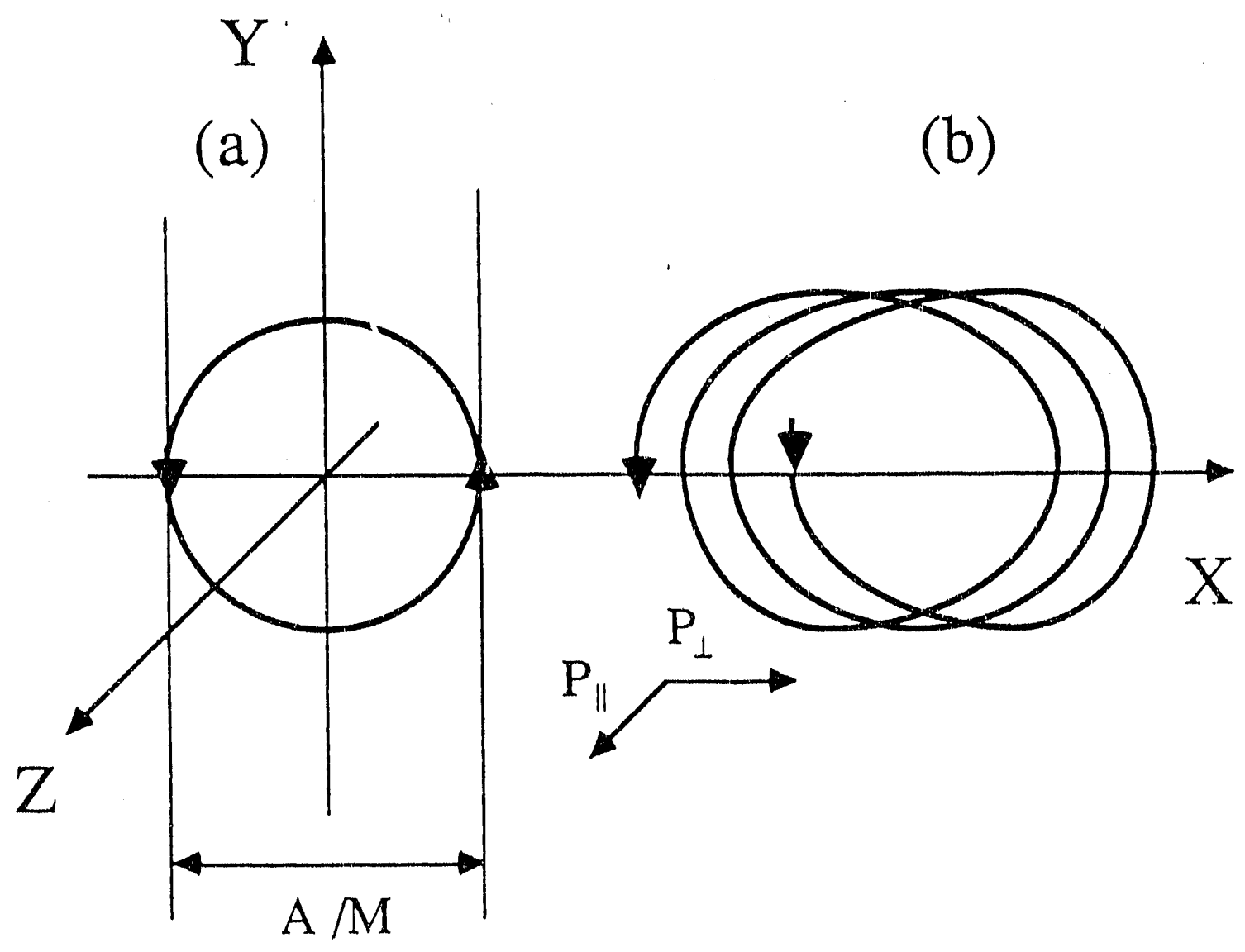

Fig.2 


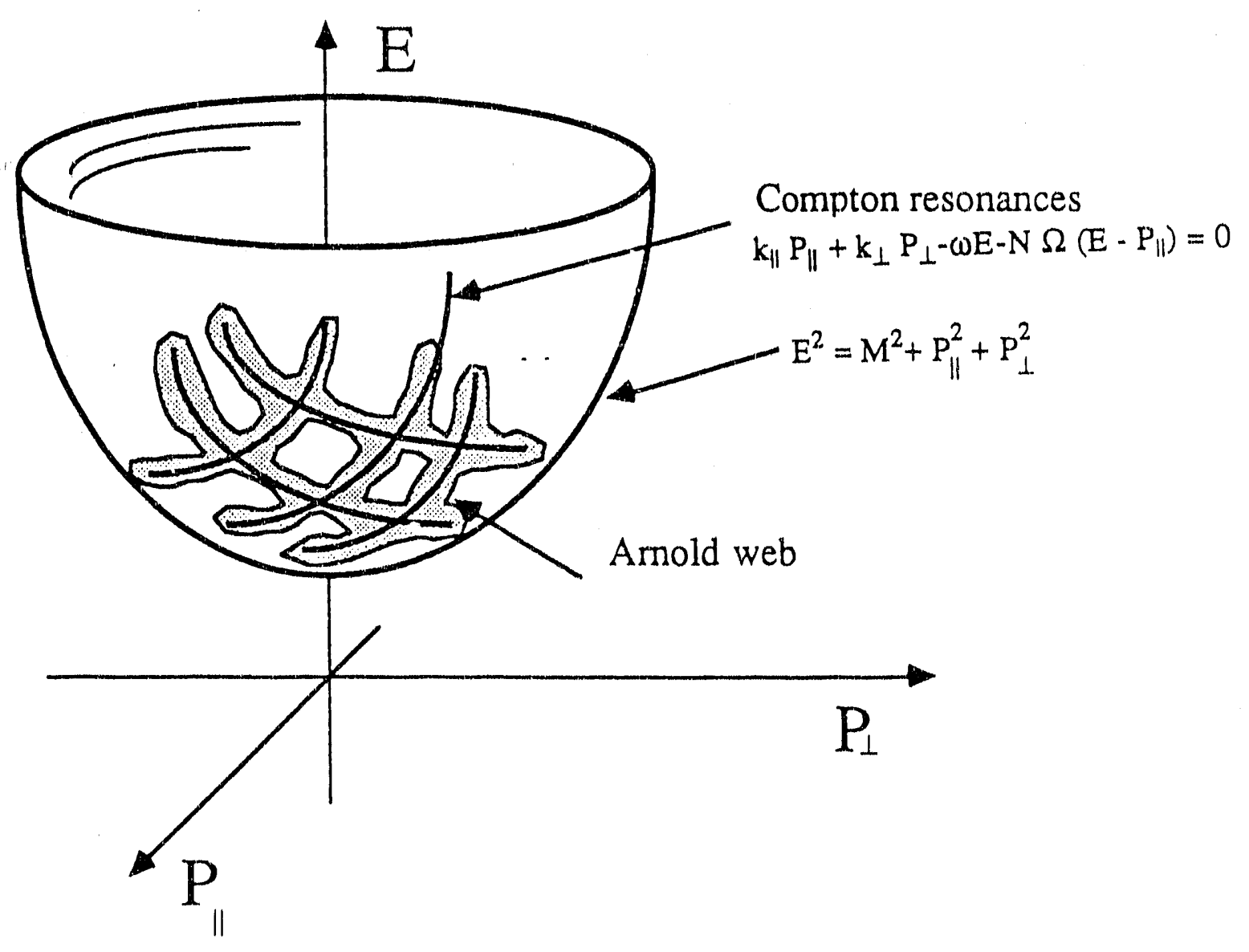

Fig. 3 


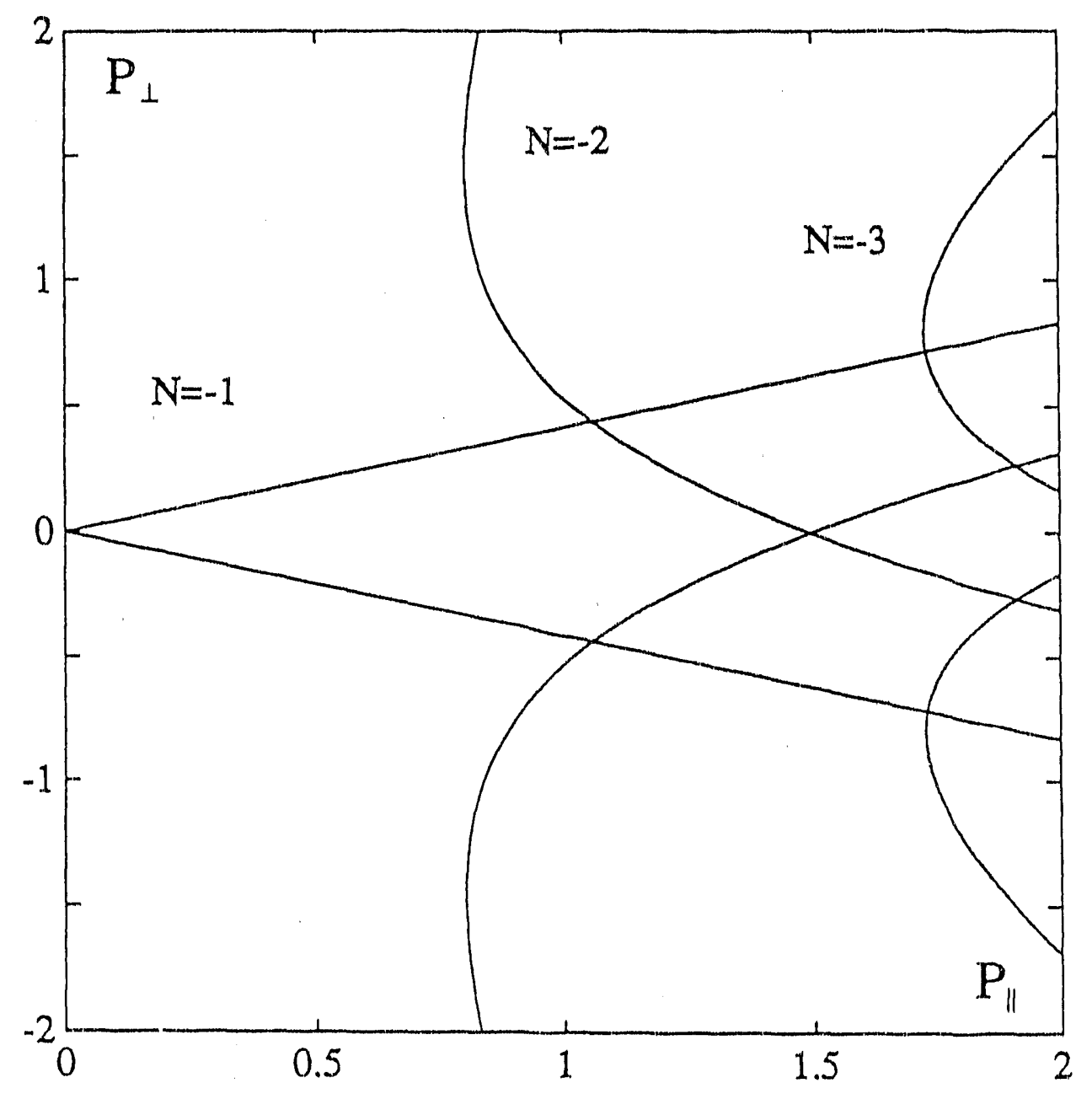

Fig.4 


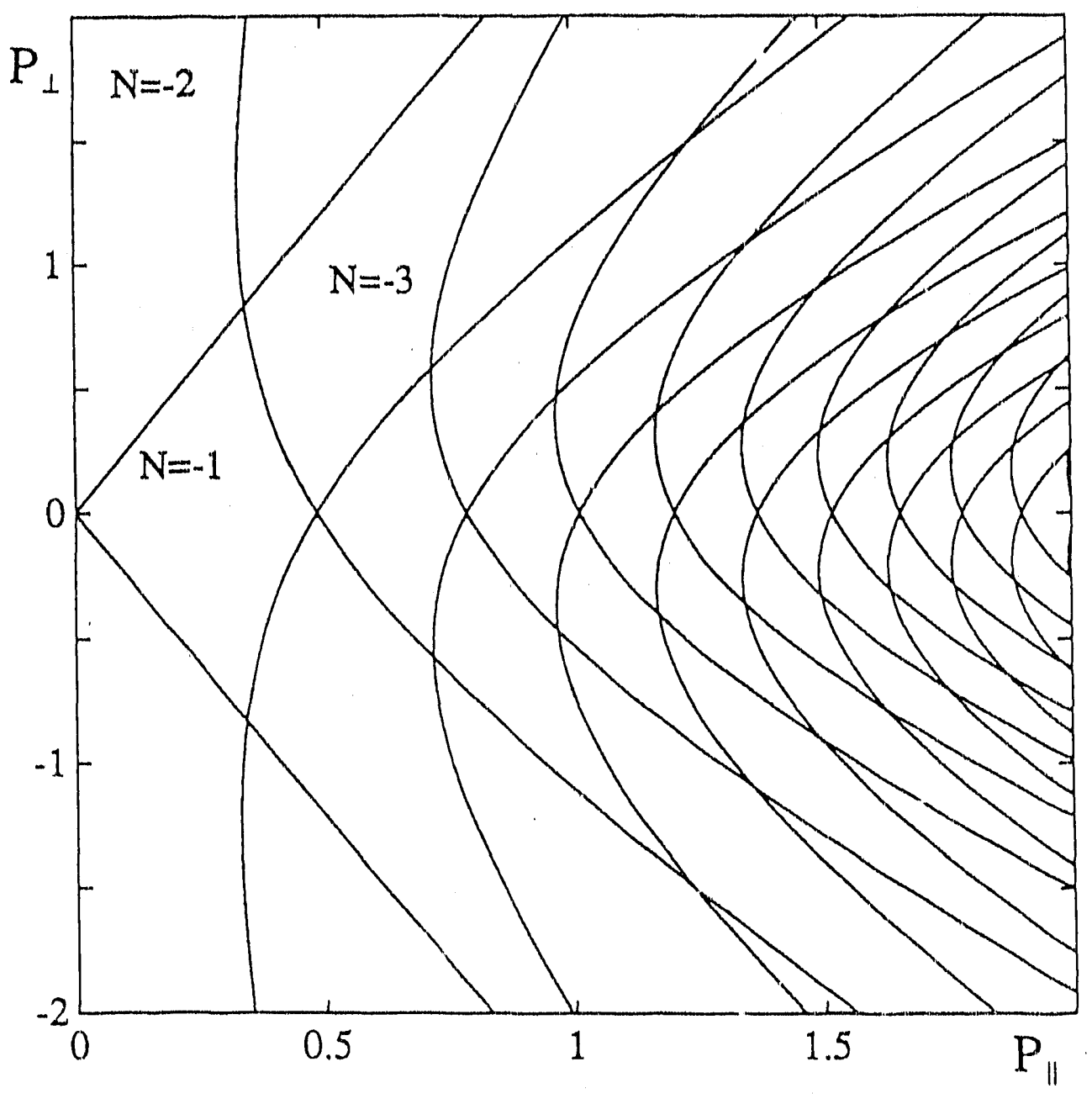

Fig.5 
Dr. F. Peoton, Univ, of Wollonging, AUSTFULLA

Prol. M.H. Brennan, Univ, of Syoney, AUSTRALIA

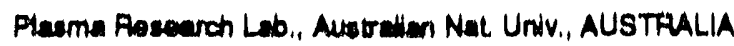

Prot, I.A. Jones, Finders Univ, AUSTRALLA

Prot. F. Cep, Inst. for Theoretral Physics, AUSTRIA

Prol. M. Hoindier, Inattut for Theoretseche Phyalk, AUSTAIA

Prot. M. Gooscons, Aetronomisch Inettuut, BELGIUM

Ecolu Royde NHHidro, Lab. de Phy, Plasmas, BELQIUM

Commiation-Europeen, DG. XII-Fusion Prod, BELGIUM

Prol. R. Boudiquh, Rikeuniverd Gent, BELGIUM

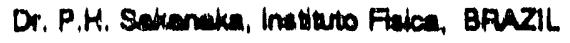

Inathus Neciond Do Poncuisas Espadele-INPE, BRAZIL

Documents Orito, Atomic Energy of Cenede Lid., CANAOA

Dr. M.P. Bectynetei, APB Technologies, Inc., CANADA

Dr. H.M. Skarsoard, Univ. of Saktukchewren, CANADA

Prol. J. Tolchmem, Univ, of Montred, CANADA

Prol. S.R. Sreonivaem, Univ. or Celgery, CANADA

Prof. T.W. Jotnetion, INRS-Energia, CAMADA

Dr. R. Bolion, Contre canadien de fution meondeque, CANADA

Dr. C.R. Jemes." Univ. of Aberta, CANADA

Dr. P. Lukle, Komenetcho Untworsita, CZECHOSLOVAKIA

The LUbrarian, Cutham Leboratory, ENGLAND

Litbrery, A61, Puthertord Apploton Leboratory, ENGLAND

Mrs. S.A. Hutchinwon, JET Librory, ENGLAND

Dr. S.C. Shurma, Univ. oi South Pecific, FIJI ISLANDS

P. Mahomen, Univ. of Holeinid, FINLAND

Prot. M.N. Busece, Ecole Potymatinique, FRANCE.

C. Mouthed, Lob. Phycique des Miloux loniste, FPANCE

J. Ractal, CENCADAPACHE - Bar 506, FPANCE

Prof. E. Economou, Univ. of Cren, GREECE

Ms. C. Pinni, Univ. of loannina, GAEECE

Dr. T. Mud, Academy Buthographio Ser., HONG KONG

Proprint Lubrary, Hungarian Acadorny of SCI., HUNGARY

Dr. B. Ons Gupta, Saha inst of Nuctoer Phyeics, INDIA

Dr. P. Kow, Inst. Lo Plesma Research, INDIA

Dr. P. Rocenaw, lerad inst. of Technology, ISPAEL.

Lbrerian, International Convar for Theo Phyzics, ITALY

Miss C. Do Pab, Ascociazion EURATOA4ENEA, ITALY

D. G. Groseo, letituto of Fiava dad Plasna, ITALY

Prof. G. Rostangni, Istituro Gas lonkzzati Dol Cnr, ITALY

Dr. H. Yameto, Toshiba Rea d Devid Center, JAPAN
Prof. I. Kewnakami, Hiroshima Univ., JAPAN

Prof, K. Nlehilkawa, Hroshima Univ., JAPAN

Drroctor, Jepen Atomio Enorgy Research Inst, JAPAN

Prol, S. Itoh, Kyuahu Univ., JAPAN

Pesearch into. Ctr, Navoned instle for Fusion Sclence, JAPAN

Prol, S. Tenaka, Kyoto Univ., JAPAN

Librery, Kyoto Univ., JAPAN

Prol, N. Inowe, Univ, of Tohyo, LAPAN

Secresary, Pluma secilon, Eloctrotachnical Lab., JAPAN

S. Mon, Tectiniod Adweor, LAEPI, LAPAN

Dr. O. Murai, Kumemow mut of Technology, JAPAN

J. Hyeon-Sook, Korsen Atomic Enorgy Reseurch Inst, KOAEA

D.1. Chad, The Korean Adv, Inat. of Sod. Toch, KOAEA

Prot. B.S. Lhoy, Univ, of Welkato, NEW ZEALAND

Inst of Phyla Chinea Acad SA PEOPLE'S REP. OF CHINA

Librey, Inst of Plasma Physica, PEOPLE'S REP. OF CHINA

Toinghua Univ, LJbrary, PEOPLE'S REPUBLIC OF CHINA

2. U, S.W. ina Phydics, PEOPLE'S AEPUBUC OF CHINA

Prol. J.A.C. Cebred, Inathuto Superior Tecribo, PORTUGAL.

Dr. O. Potrus, AL I CUzA Univ, ROMMANIA

Dr. J. do Vikers, Fusion Studios, AEC, S. AFAICA

Prol. M.A. Howberg, Univ, of Natal, S. AFAICA

Prot, D.E. Km, Pohang inat. of Sol, Tech, SO. KOREA

Prot C.I.E.M.A.T, Fuaton Otviaion Librayy, SPAIN

Dr. L Stanth, UNiv. of UMEA, SWEDEN

Lbrey, Royd inct, of Technology, SWEDEN

Prot. H. Whatmeon, Chedmers Univ, of Tech., SWEDEN

Compe Phys. Dos Plaemas, Ecole Polytect, SWITZERLAND

Bibliother, Inst. Voor Ptasma-Fysica, THE NETHERLANDS

Asat. Prot. Or. S. Culder, Midde East Tech. Univ., TURKEY

Dr. V.A. Gulthikh, Sai. Res. Inst. Eloctrophys. Apparatus, USSR

Dr. D.D. Ayutov, Siberian Brench of Acadomy of Sol, USSA

Dr. G.A. Elinew, I.V. Kurchator Inst, USSA

Litrorien, The Ukr.SSA Acadorny of Sciancos, USSA

Dr. L.M. Kovrizhnimkh, Inst. of Gonorad Ptiystes, USSR

Kerntorectungeanlege GmbH, Zentrabibliothek, W. GERMANY

Bibliothek, Inst. For Plasmatoraching, W, GERMANY

Prot. K. Sctinder, Ruly-Universita Bochum, W. GERMANY

Dr. F. Wogner, (ASOEX), Nax-Planck-Institut, W. GEAMANY

Librarian, Max-Plenck.Institut, W. GERMANY

Prot. R.K. Jenew, Inst. of Ptyeice, YUGOSLAVIA 

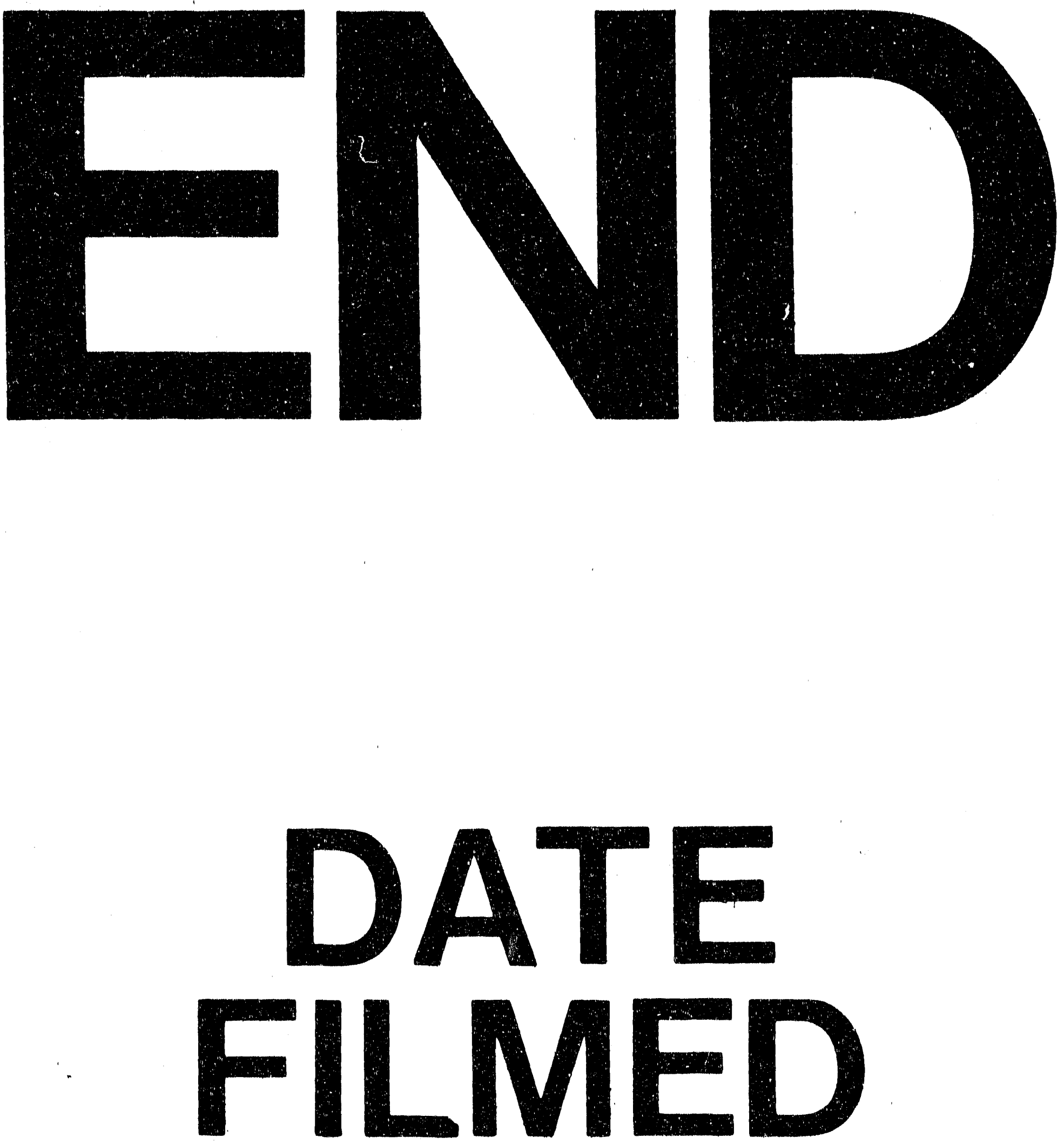

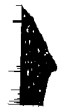

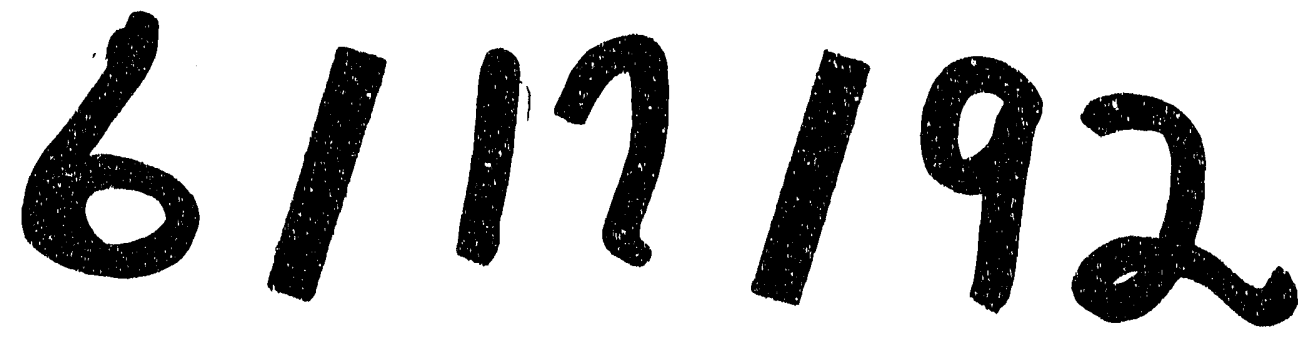


